\title{
Correction to: Reducing neonatal mortality and respiratory distress syndrome associated with preterm birth: a scoping review on the impact of antenatal corticosteroids in low- and middle-income countries
}

\author{
Stanley Mwita ${ }^{1} \cdot$ Mary Jande $^{1} \cdot$ Deogratias Katabalo $^{1} \cdot$ Benjamin Kamala $^{2,3} \cdot$ Deborah Dewey $^{4,5,6}$ \\ Published online: 23 January 2021 \\ (c) Children's Hospital, Zhejiang University School of Medicine 2021
}

\section{Correction to: World Journal of Pediatrics} https://doi.org/10.1007/s12519-020-00398-6

In the Original publication of the article, the authors found errors in the authors' affiliations. The correct representation of affiliations is listed below.

Stanley Mwita ${ }^{1}$, Mary Jande ${ }^{1}$, Deogratias Katabalo ${ }^{1}$, Benjamin Kamala ${ }^{2,3}$, Deborah Dewey ${ }^{4,5,6}$

${ }^{1}$ School of Pharmacy, Catholic University of Health and Allied Sciences, P.O. Box 1464 Bugando Area, Mwanza, Tanzania

${ }^{2}$ Department of Research, Haydom Lutheran Hospital, P.O. Box 9000, Haydom, Manyara, Tanzania

The original article can be found online at https://doi.org/10.1007/ s12519-020-00398-6.

Stanley Mwita

stanleymwita@gmail.com

1 School of Pharmacy, Catholic University of Health and Allied Sciences, P.O. Box 1464, Bugando Area, Mwanza, Tanzania

2 Department of Research, Haydom Lutheran Hospital, P.O. Box 9000, Haydom, Manyara, Tanzania

3 Department of Epidemiology and Biostastitics, School of Public Health and Social Sciences, Muhimbili University of Health and Allied Sciences (MUHAS), P.O. Box 65001, Dar es Salaam, Tanzania

4 Alberta Children's Hospital Research Institute, Alberta Children's Hospital, 2888 Shaganappi Trail NW, Calgary, AB T3B 6A8, Canada

5 Department of Pediatrics, University of Calgary, 28 Oki Dr NW, Calgary, AB T3B 6A8, Canada

6 Department of Community Health Sciences, University of Calgary, 3D10, 3280 Hospital Drive NW, Calgary, AB T2N 4Z6, Canada
${ }^{3}$ Department of Epidemiology and Biostatics, School of Public Health and Social Sciences, Muhimbili University of Health and Allied Sciences (MUHAS), P.O. Box 65001, Dar es Salaam, Tanzania

${ }^{4}$ Alberta Children's Hospital Research Institute, Alberta Children's Hospital, 2888 Shaganappi Trail NW, Calgary, AB, T3B 6A8, Canada

${ }^{5}$ Department of Pediatrics, University of Calgary, 2888 Shaganappi Trail NW, Calgary, AB, T3B 6A8, Canada

${ }^{6}$ Department of Community Health Sciences, University of Calgary, 3D10, 3280 Hospital Drive NW, Calgary, AB, T2N 4Z6, Canada 